\title{
Expression of Ki-67 and Its Association with Histological Type, Grade and Stage of Colorectal Carcinoma
}

\author{
Sultana Israt Jahan', Shah Md. Badruddoza', S. M. Asafudullah'3 , Md. Nurul Amin ${ }^{4}$
}

\section{ABSTRACT}

Background \& objective: The role Ki-67 as a prognostic marker has been studied in many cancers in many studies. However, only few studies reported the prognostic role of Ki-67 in colorectal carcinoma (CRC) with contradictory opinions. The present study was undertaken to assess the Ki-67 proliferation index (PI) in tissue sections of CRC and to investigate the relationship between the proliferative activity and histological type, grade and stage of the tumour.

Methods: The present cross-sectional descriptive study was conducted in the Department of Pathology, Rajshahi Medical College, Rajshahi over a period of two years from September 2017 to August 2019. All clinically suspected cases of CRC and later confirmed by histopathology were the study population. A total 44 such cases were included in the study. Tissue sections from 44 formalin-fixed and paraffin-embedded tumor specimens were examined at the Department of Pathology of Rajshahi Medical College, Rajshahi. The cases were histologically classified, graded (WHO) and staged according to TNM and modified Dukes' staging system. Ki-67 proliferation index was calculated immunohistochemically using the monoclonal antibody MIB-1, and were studied under light microscope. Expression of Ki-67 was calculated as a percentage of labeled nuclei per 500 cells counted in consecutive five high-power fields in the most reactive areas of the slides. The staining results were categorized into groups using a cut-off value of Ki- 67 determined by ROC curve analysis.

Results: In the present study the cases with high Ki-67 PI were significantly associated with high grade colorectal carcinoma ( $p=0.005)$. TNM Stage-II and Stage-III tumours were more likely to be associated with high Ki-67 PI than those with low Ki-67 PI $(p<0.001)$. A significant association was observed between Ki-67 immuno-expression and modified Dukes' staging of the tumours with Stage $C$ being highly associated with high Ki-67 PI $(p<0.001)$. But histologic tumor type (mucinous and non-mucinous) was not found to be associated with Ki-67 proliferation index. Based on Receiver Operating Characteristic (ROC) curve analysis tumors with nuclear immunoreactivity $\geq 25 \%$ were considered as high proliferation index (PI) and $<25 \%$ as low PI. The Ki-67 at a cut-off value of $>25 \%$ had high sensitivity $(85.7 \%)$ in differentiating high grade CRC from the low-grade tumors but its specificity was moderate $(67.6 \%)$ with overall diagnostic accuracy being $70.5 \%$.

Conclusion: The study concluded that cases with high Ki-67 PI are significantly associated with high grade CRC (poorly differentiated) than the cases with low grade CRC (well and moderately differentiated). TNM Stage-II \& Stage-III and modified Dukes' stage C also more often tend to be associated with high Ki-67 PI than with low Ki-67 PI.

Key words: Colorectal carcinoma, Ki-67 proliferation activity, histologic grade, stage and type of tumours, sensitivity, specificity etc.

\section{Author's information:}

'Dr. Sultana Israt Jahan, MBBS, MD (Pathology), Medical officer, Upozilla Health Complex, Godagari, Rajshahi. Ex Resident MD (Pathology), Department of Pathology, Rajshahi Medical College, Rajshahi

${ }_{2}^{2}$ Prof. Dr. Shah Md. Badruddoza, MBBS, FCPS (Histopathology), Ex Professor and Head Department of Pathology, Rajshahi Medical College, Rajshahi

${ }^{3}$ Prof. Dr. S. M. Asafudullah, MBBS, MD (Pathology), Professor and Head, Department of Pathology, Rajshahi Medical College, Rajshahi ${ }^{4}$ Dr. Md. Nurul Amin, Associate Professor (Research \& Development) \& Executive Editor, Ibrahim Cardiac Medical Journal, Ibrahim Cardiac Hospital \& Research Institute, Shahbag, Dhaka.

Correspondence: Dr. Sultana Israt Jahan, Cell Phone: +8801722612241 Email:dr.sijahan@gmail.com 


\section{INTRODUCTION:}

Colorectal cancer (CRC) is the third most common cancer in men (10 percent of total cancers in men), and the second most common in women worldwide (9.2 percent of total cancers in women). In more developed regions, approximately $55 \%$ of cases occur. There is wide geographical variation in the incidence of CRC around the world, but the geographical pattern in men and women is quite similar all over the world. ${ }^{1}$ In Bangladesh, $6.5 \%$ and $2.5 \%$ of all cancers in males and females respectively are CRCs. ${ }^{2}$

Histopathological examination is the gold standard diagnostic approach for CRC, and the strongest prognostic factors are the type, grade and stage of the tumour. However, the prognosis among individual patients with CRC at an equivalent stage is very heterogeneous. In this context, improved prognosis and patient survival can be achieved by using immunohistochemistry studies with proliferation marker Ki-67, a proliferating antigen in the nuclear cell. ${ }^{3}$ Malignant tumor growth is highly variable, and this probably reflects the direction of their clinical course. However, proliferation is a key feature of progression of tumour. $\mathrm{Ki}-67$ is a specific marker for cell proliferation and is a proliferation- associated nuclear antigen that is expressed in all cycling cells except GO resting cells, and represents cells in particular in the $\mathrm{S} / \mathrm{G} 2+\mathrm{M}$ phase. MIB-1 is a monoclonal antibody, which recognizes Ki-67 nuclear antigen in the formalin-fixed paraffin embedded tissue sections. The expression of $\mathrm{Ki}-67$ is calculated as the percentage of tumor cells positively stained by the antibody, with the most specific criterion of positivity being nuclear staining. ${ }^{4}$

The quantification of $\mathrm{Ki}-67$ expression by Immunohistochemistry (IHC), known as Ki-67 proliferation index (PI), is done to estimate the growth fraction of tumour. A marked variation in the amount of Ki-67 expression within different types, grades and stages of same tumour is seen indicating that Ki-67 staining is useful in the assessment of individual tumour prognosis. The role of $\mathrm{Ki}-67$ has not been established in determining the prognosis of GIT cancer yet. Only few studies exist on the prognostic role of $\mathrm{Ki}-67$ in colorectal carcinoma. This study was designed to evaluate the Ki-67 expression in colorectal carcinoma (in terms of $\mathrm{Ki}-67$ proliferation index (PI) in tissue sections) and to investigate the relationship between the proliferative activity and histological type, grade and stage of the tumour. The findings derived from the study would help in choosing proper management option in a particular tumour for better outcome.

\section{METHODS:}

This cross-sectional analytical study was carried out in the Department of Pathology, Rajshahi Medical College (RMC), Rajshahi in collaboration with the Department of Surgery, RMC Hospital, Rajshahi and Armed Forces Medical College, Dhaka Cantonment, Dhaka over a period of two years from September 2017 to August 2019. A total of 44 resected specimens of CRC, confirmed by histopathological examination, were included in the study. Tissue processing and staining were done according to standard protocol followed at Rajshahi Medical College. Sections were studied under light microscope and classified microscopically. The cases were histologically graded and staged according to TNM and modified Dukes' staging system.

Immunohistochemistry was done in Armed Forces Institute of Pathology (AFIP), Dhaka. For immunohistochemistry, 3-4 micrometer thick sections of formalin fixed, paraffin-embedded tissues were used. The sections were incubated with a MIB-1 monoclonal antibody in appropriate dilutions. Standard immunohistochemical method was applied for subsequent staining. Brown nuclear staining was considered positive for Ki-67. The positive nuclear staining was observed in the epithelial cells of normal colonic mucosa and in the lymphoid cells and they served as internal positive control. Expression of Ki-67 was calculated as a percentage of labeled nuclei per 500 cells counted in consecutive five high-power fields in the most reactive areas of the slides. Cells with questionable nuclear staining were discounted. Necrotic or thick areas and severely overlapping tumour cells were avoided during evaluation. The staining results were categorized into groups according to the percentage of $\mathrm{Ki}-67$ positive tumor cells and the cut-off value for categorization was determined by ROC curve analysis. 
All statistical analyses were performed using the Statistical Package for Social Science (SPSS), version 20 for windows. Associations of $\mathrm{Ki}-67$ with histopathologic type, grade, and stage were determined with the help of Chi-square $\left(\chi^{2}\right)$ Test. The level of significance was set at $5 \%$ and $p$-value $<0.05$ was considered significant. Accuracy of the $\mathrm{Ki}-67$ in grading CRC was determined by comparing the findings of the $\mathrm{Ki}-67$ with those of histopathology. The components of accuracy test (sensitivity, specificity, positive and negative predictive values) were computed by respective formulae for the tests.

\section{RESULTS:}

\section{Demographic characteristics:}

The predominant age group was 40 - 60 years $(63.6 \%)$ followed by $<40$ years $(25 \%)$ and 60 or $>60$ years $(11.4 \%)$. The mean age of the study subjects was $46.3 \pm 12$ (range: $21-80$ ) years. Fifty percent were male and $50 \%$ female (table I).

\section{Morphological/Anatomical characteristics:}

The median size of the tumour was $20.0 \pm 2.7 \mathrm{~cm}$ with smallest and largest tumours being 2 and $80 \mathrm{~cm}$ respectively. Nearly one-third (31.8\%) of the tumours was located in left colon followed by $27.3 \%$ in right colon, $25 \%$ in transverse colon and $15.9 \%$ in rectum (table II).

\section{Histopathological characteristics:}

In terms of differentiation, $25 \%$ were welldifferentiated, $59.1 \%$ moderately differentiated and $15.9 \%$ poorly differentiated. Over one-third (36.4\%) were mucinous type and $63.6 \%$ were non-mucinous type. Over half (56.8\%) was at modified Dukes' stage B and $42.2 \%$ were at stage C. TNM staging of the tumour shows that $45.5 \%$ were at Stage-I, $11.3 \%$ at stage-II and $43.2 \%$ at Stage-III (Table III).

\section{Determining cut-off value of $\mathbf{K i}-67$ in predicting high grade colorectal carcinoma:}

Before predicting the accuracy of $\mathrm{Ki}-67$ in differentiating high grade from low-grade CRCs, an optimum cut-off value for $\mathrm{Ki}-67$ was determined using a Receiver Operating Characteristic (ROC) (Fig.1). The best cut-off value for high sensitivity without much compromise with specificity obtained from the tables IV \& V was 22.5 with an area under the curve being $0.780[(95 \% \quad C I=0.581-0.979)$, $p=0.020]$. The area under the curve indicates that $78 \%$ of the high-grade tumours could be correctly diagnosed with Ki-67 PI $22.5 \%$ or more in CRC patients. As in the present series there was no other value of Ki- 67 between 22.5 and 27.5 but 25, it was taken as the best cut-off value for predicting high grade CRCs.

\section{Distribution of Ki-67 among CRC patients:}

Based on the ROC curve analysis, the test variable $\mathrm{Ki}-67$, was divided into two groups. Ki-67 $\geq 25 \%$ were considered as high proliferation index (PI) and $<25 \%$ were considered as low PI. Accordingly, $26(59.1 \%$ ) subjects had Ki-67 < 25\% (low PI) and $18(40.9 \%$ ) had $>\mathrm{Ki}-67 \geq 25 \%$ (high PI) (Table VI).

\section{Association of Ki-67 PI with grading, staging and histologic type of the CRCs:}

Over $60 \%$ of the cases with high Ki-67 PI (Ki-67 PI $\geq 25 \%$ ) had moderately differentiated (G2) and $33.3 \%$ had poorly differentiated tumours (G3) as compared to $57.7 \%$ and $3.8 \%$ respectively of the cases with low Ki-67 PI (Ki-67 PI < 25\%. The cases with high Ki-67 PI were significantly associated with high grade colorectal carcinoma (poorly differentiated) than the cases with low Ki-67 PI $(p=0.005)$. No significant difference was observed between $\mathrm{Ki}-67$ immuno-expression and histologic type of the tumours, although mucinous type of tumours was a bit higher in cases with high Ki-67 PI ( $p=0.772)$. A significant association was observed between high Ki-67 PI and modified Dukes' staging of the tumours with Stage $C$ being frequently associated with high Ki-67 PI cases ( $p<0.001)$. Association between TNM staging and Ki-67 PI shows that Stage-II \& Stage-III tumours tend to be associated with high $\mathrm{Ki}-67$ PI more often than those with low Ki-67 PI ( $p$ $<0.001$ ) (table VII).

\section{Accuracy of Ki-67 in differentiating high grade CRC from the low grade:}

The accuracy of Ki-67 in diagnosing high grade CRC is shown in table VIII. The sensitivity of the test in differentiating high grade CRC from the low-grade 
tumours was $85.7 \%$ and specificity of the test in correctly detecting those who were low grade (welland moderately-differentiated) was $67.6 \%$. The positive and negative predictive values of the test were $33.3 \%$ and $96.2 \%$ respectively, while the percentages of false positive and false negatives were $66.7 \%$ and $3.8 \%$ respectively. The overall diagnostic accuracy was $70.5 \%$.

Table I. Distribution of cases by Demographic characteristics ( $n=44)$

$\begin{array}{lcc}\text { Demographics } & \text { Frequency } & \text { Percentage } \\ \begin{array}{l}\text { Age* (years) } \\ <40\end{array} & 11 & \\ 4060 & 28 & 25.0 \\ \geq 60 & 05 & 63.6 \\ \text { Gender } & & 11.4 \\ \text { Male } & 22 & 50.0 \\ \text { Female } & 22 & 50.0\end{array}$

${ }^{*}$ Mean age $=46.3 \pm 12.1$ years; range $=21-80$ years .

Table II. Distribution of cases by morphological/anatomical characteristics $(n=44)$

\begin{tabular}{|c|c|c|c|}
\hline $\begin{array}{l}\text { Anatomical } \\
\text { characteristics }\end{array}$ & Frequency & Percentage & $\begin{array}{l}\text { Median } \pm \text { SEM } \\
\quad \text { (range) }\end{array}$ \\
\hline Tumor size $(\mathrm{cm})$ & -- & -- & $20.0 \pm 2.7(2-80)$ \\
\hline \multicolumn{4}{|l|}{ Location / Site } \\
\hline Right colon & 12 & 27.3 & -- \\
\hline Transverse colon & 11 & 25.0 & -- \\
\hline Left colon & 14 & 31.8 & -- \\
\hline Rectum & 7 & 15.9 & -- \\
\hline
\end{tabular}

${ }^{*}$ Mean age $=46.3 \pm 12.1$ years; range $=21-80$ years .

Table III. Distribution of cases by histopathological characteristics $(n=44)$

\begin{tabular}{lcc|} 
Histopathological characteristics & Frequency & Percentage \\
$\begin{array}{l}\text { Differentiation } \\
\text { Well (G1) }\end{array}$ & 11 & 25.0 \\
Moderate (G2) & 26 & 59.1 \\
$\quad$ Poor (G3) & 7 & 15.9 \\
Histologic type & & \\
$\quad$ Mucinous adenocarcinoma & 16 & 36.4 \\
$\quad$ Non-mucinous adenocarcinoma & 28 & 63.6 \\
Modified Duke's staging & & \\
B & 25 & 56.8 \\
C & 19 & 43.2 \\
TNM staging & & \\
Stage-I & 20 & 45.5 \\
Stage-II & 05 & 11.3 \\
Stage-III & 19 & 43.2
\end{tabular}

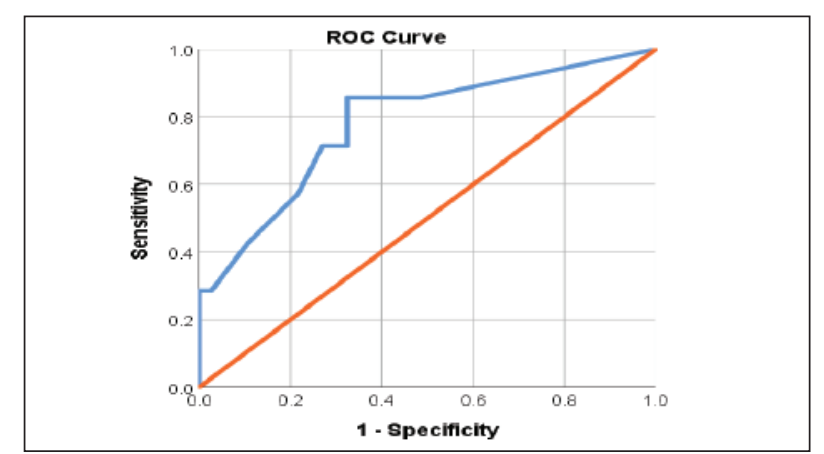

Fig. 1 showing area under the ROC curve

\begin{tabular}{|c|c|c|c|c|}
\hline \multicolumn{5}{|c|}{ Test Result Variable(s): Ki-67 } \\
\hline \multirow[b]{2}{*}{ Area } & \multirow[b]{2}{*}{ Std. Error ${ }^{\mathrm{a}}$} & \multirow[b]{2}{*}{$p$-value ${ }^{b}$} & \multicolumn{2}{|c|}{$\begin{array}{l}95 \% \text { Confidence Interval of } \\
\text { Area under the Curve }\end{array}$} \\
\hline & & & Lower Bound & Upper Bound \\
\hline 0.780 & 0.101 & 0.020 & 0.581 & 0.979 \\
\hline
\end{tabular}

a. Under the nonparametric assumption

b. Null hypothesis: true area $=0.5$

\begin{tabular}{|c|c|c|}
\hline \multicolumn{3}{|c|}{ Test Result Variable(s): Ki-67 } \\
\hline $\begin{array}{l}\text { Positive if Greater } \\
\text { Than or Equal To }\end{array}$ & Sensitivity & 1-Specificity \\
\hline 4.00 & 1.000 & 1.000 \\
\hline 7.50 & 0.857 & 0.486 \\
\hline 12.50 & 0.857 & 0.459 \\
\hline 17.50 & 0.857 & 0.432 \\
\hline 22.50 & 0.857 & 0.324 \\
\hline 27.50 & 0.714 & 0.324 \\
\hline 35.00 & 0.714 & 0.270 \\
\hline 45.00 & 0.571 & 0.216 \\
\hline 55.00 & 0.429 & 0.108 \\
\hline 65.00 & 0.286 & 0.027 \\
\hline 72.50 & 0.286 & 0.000 \\
\hline 77.50 & 0.143 & 0.000 \\
\hline 81.00 & 0.000 & 0.000 \\
\hline
\end{tabular}

The test result variable(s): $\mathrm{Ki}-67$ has at least one tie between the positive actual state group and the negative actual state group. a. The smallest cut-off value is the minimum observed test value minus 1 , and the largest cut-off value is the maximum observed test value plus

1. All the other cut-off values are the averages of two consecutive ordered observed test values.

TableVI. Distribution of Ki-67 among CRC patients $(n=44)$

$\begin{array}{lcc}\mathrm{Ki}-67^{*}(\%) & \text { Frequency } & \text { Percentage } \\ \geq 25 & 18 & 40.9 \\ <25 & 26 & 59.1\end{array}$




\begin{tabular}{lccc}
\hline Table VII: Association between histologic characteristics and Ki-67 PI \\
\cline { 2 - 3 } $\begin{array}{l}\text { Histologic } \\
\text { characteristics }\end{array}$ & $\begin{array}{c}\text { Ki-67 (\%) } \\
(\mathrm{n}=18)\end{array}$ & $\begin{array}{c}\text { Low PI }<25) \\
(\mathrm{n}=26)\end{array}$ & p-value \\
Differentiation & & & \\
Well (G1) & $1(5.6)$ & $10(38.5)$ & \\
Moderate (G2) & $11(61.1)$ & $15(57.7)$ & 0.005 \\
Poor (G3) & $6(33.3)$ & $1(3.8)$ & \\
Histologic type & & & \\
Mucinous & $7(38.9)$ & $9(34.6)$ & 0.772 \\
Non-mucinous & $11(61.1)$ & $17(65.4)$ & \\
Modified Dukes' staging & & & \\
B & $4(22.2)$ & $21(80.8)$ & $<0.001$ \\
C & $14(78.8)$ & $5(19.2)$ & \\
TNM Staging & & & \\
Stage-I & $1(5.6)$ & $19(73.1)$ & \\
Stage-II & $3(16.7)$ & $2(7.7)$ & $<0.001$ \\
Stage-III & $14(77.7)$ & $5(19.2)$ &
\end{tabular}

*Data were analyzed using Chi-square $\left(\chi^{2}\right)$ Test.

Figures in the parentheses denote corresponding percentage.

\begin{tabular}{|lc|}
\hline Table VIII: Distribution of patients by diagnostic accuracy $(\mathbf{n}=\mathbf{4 4})$ \\
\hline Accuracy test components & Percentage \\
\hline Sensitivity & 85.7 \\
Specificity & 67.6 \\
PPV & 33.3 \\
NPV & 96.2 \\
$\%$ false +ve & 66.7 \\
\% of false -ve & 3.8 \\
Diagnostic accuracy & 70.5
\end{tabular}

\section{Discussion:}

Colorectal carcinoma is a major cause of morbidity and mortality worldwide. ${ }^{5}$ Cellular proliferation is fundamental to maintain tissue homeostasis and is important in oncogenesis. More recently, advances in understanding tumour biology have led to the development of targeted therapy, allowing progress in the treatment of $\mathrm{CRC}^{6}$ and quantification of cell proliferative activity in neoplasia is currently the subject of considerable interest as assessment of tumour cell proliferation may predict tumour behaviour. ${ }^{7}$ The $\mathrm{Ki}-67$ is a nuclear antigen expressed in highest concentration in all stages of the cell cycle, but not in resting cells and it is widely used in routine pathology as a proliferation marker to measure the growth fraction in human tumours. MIB-1 is a monoclonal antibody that recognizes a fixation resistant epitope of Ki-67 antigen; and has allowed retrospective examination and estimation of the proliferative fraction of neoplasia. ${ }^{8}$ This study was designed to evaluate the immune-expression of $\mathrm{Ki}-67 \mathrm{PI}$ in formalin-fixed, paraffin-embedded tissue sections of colorectal carcinomas and to investigate the relationship between the proliferative activity of colorectal carcinoma with histological type, grade, and stage of the tumour.

In the present study the cases with high $\mathrm{Ki}-67$ proliferation index were significantly associated with high grade colorectal carcinoma than the cases with low grade CRC $(p=0.005)$. Advanced stage tumours (TNM Stage-II \& Stage-III and modified Dukes' Stage $C$ ) tend to be associated with high Ki-67 PI more often than those with low Ki-67 PI ( $p$ $<0.001$ ). But histologic type of tumour was not found to be associated with $\mathrm{Ki}-67$ proliferation index. Similar findings were reported by other studies $^{8,9}$ in which the Ki-67 PI appeared to increase with decreasing degree of differentiation of carcinoma. Another highly significant relation observed between Ki-67 PI and histological type of CRC $(p=0.005)$, in which the proliferative activity was higher in non-mucinous tumours than in mucinous and signet ring carcinoma, which contrasts with the findings of the present study. The reverse finding is also reported by some other studies. Ahmed and colleagues $^{10}$ found over-expression of $\mathrm{Ki}-67$ proliferation protein in $62 \%$ cases with significant associations between Ki-67 proliferation index and histologic type $(p=$ $0.005)$ and tumour grade $(p=0.018)$; but no significant relation was observed with the other clinicopathological parameters such as age, gender, tumour's size, site, depth, stage, nodal status, and vascular invasion $(p>0.05) \cdot{ }^{10}$ The findings obtained from Ahmed's study indicate that proliferating index was low in poorly differentiated tumours (Grade III) than well or moderately differentiated carcinomas (Grade I and Grade II). This is in contrast with the findings of the present study. A similar study from Japan, ${ }^{11}$ also demonstrated that the positive rate of Ki-67 PI in poorly differentiated adenocarcinoma and mucinous carcinomas was significantly lower than in well differentiated and moderately 
differentiated adenocarcinomas, suggesting that proliferative activity is low in cancers with poor differentiation.

Worldwide, CRC showed a wide range of $\mathrm{Ki}-67 \mathrm{PI}$, ranging from $13-90 \%,{ }^{7-9,11}$ indicating a variation in proliferative activity. Explanation for this wide-ranged variation in the proliferative activities of CRC, as measured by MIB-1 antibody, among studies could be due to a difference in epitope preservation, in staining procedures, in methods of evaluation or quantification of Ki-67 immunostaining and in study population. Lanza et $\mathrm{al}^{12}$ showed mucinous carcinoma exhibit higher levels of Ki-67 reactivity than non-mucinous adenocarcinomas. Also, it was observed that Ki-67 PI was significantly related with the advanced TNM tumour-stage which is consistent with findings of the present study. Other studies showed that tumours in advanced stage with subserosa or deeper invasion have a low Ki-67 proliferating index than tumours in an early invasive stage, ${ }^{11}$ while some investigators showed the reverse. $^{8}$ This is supported by several studies, ${ }^{13-15}$ who reported no relation between Ki-67 immunoreactivity and various clinicopathological and prognostic variables in cases of colorectal carcinomas.

Finally, a well-designed study with large sample size $(n=1800)$ showed high Ki-67 expression to associate with low tumour stage $(p<0.0001)$ and nodal status ( $p=0.031)$, but not with tumour grade $(p=0.863)$, histological tumour type $(p=0.154)$ or tumour localisation, and was an independent prognosticator of favourable survival $(p=0.012) .{ }^{16}$ These differences in relationship of $\mathrm{Ki}-67$ with CRC grade, type and stage might be due to considerable heterogeneity in colorectal carcinomas ${ }^{17,18}$ and differences in sample size. Therefore, the findings of the present study should be interpreted with caution, particularly in case of generalization, as the study was done on a small sample size.

Summarizing the findings of the present study and discussion thereof, it is evident that immunohistochemical technique for detecting Ki-67 PI is simple and applicable to surgical specimens and reproducibility with MIB-1 antibody immunostaining is excellent even when paraffin embedded tissue sections are used. Ki-67 immune overe xpression is a frequent finding in CRC cases, but it is not enough to monitor $\mathrm{Ki}-67$ proliferation index alone for prognosis in colorectal cancer as it was not significantly related to variable clinicopathologic parameters apart from histological stage and grade of tumour.

\section{CONCLUSION:}

From the results of the study, it can be concluded that the CRC patients with high $\mathrm{Ki}-67 \mathrm{PI}$ were significantly associated with high grade colorectal carcinoma (poorly differentiated) than the subjects with low Ki-67 PI. Advanced stage tumours (TNM Stage-II \& Stage-III and modified Dukes' Stage C) also more often demonstrates its association with high Ki-67 PI than with low Ki-67 PI. But histologic type (mucinous and non-mucinous) tumor was not found to be associated with $\mathrm{Ki}-67$ proliferation index.

As the present study data show that high Ki-67 PI in CRCs is associated with worse clinical outcome, it's over expression seems to indicate a cellular state of high proliferative activity. These findings strongly argue in favour of it's clinical utilization as an independent prognostic biomarker that may contribute to the prognostic evaluation of patients with CRC and can also be used as a potential therapeutic target for cancer therapy. Ki-67 immunohistochemistry may have a clinical application in the selection of patients with colorectal cancers who might benefit from radiotherapy and/or chemotherapy. Blocking of Ki-67 either by microinjection of antibodies or through the use of antisense oligonucleotides leads to the arrest of cell proliferation. Specifically, antisense oligonucleotides and antibodies against $\mathrm{Ki}-67$ have been shown to inhibit the progression of the cell cycle.

\section{REFERENCES:}

1. Ferlay J, Soerjomataram I, Ervik M, et al. GLOBOCAN 2012 v1.0, Cancer Incidence and Mortality Worldwide: IARC Cancer Base No. 11. Lyon, France: International Agency for Research on Cancer, 2013.

2. Hussain SA, Sullivan R. Cancer control in Bangladesh. Jpn J Clin Oncol 2013;43(12):1159-69. doi: 10.1093/jjco/ hyt140. Epub 2013 Oct 25. 
3. Bhagyalaksmi A. KI-67 proliferation index and clinicopathological patterns in colorectal carcinomas. Journal of Clinical \& Scientific Research 2015;4:119-28.

4. Nabi U, Nagi AH, Sami W. Ki-67 proliferating index and histological grade, type and stage of colorectal carcinoma. J Ayub Med Coll Abbottabad 2008;20:44-48.

5. Al-Kuraya KS. KRAS and TP53 mutations in colorectal carcinoma. Saudi Journal of Gastroenterology 2009;15:4.

6. Khamly K, Jefford M, Michael M, Zalcberg J. Beyond 5fluorouracil: new horizons in systemic therapy for advanced colorectal cancer. Expert Opin Investig Drugs $2005 ; 14: 607628$.

7. Valera V, Yokoyama N, Walter B, Okamoto $H$, Suda $T$, Hatakeyama K. Clinical significance of Ki-67 proliferation index in disease progression and prognosis of patients with resected colorectal carcinoma. Br J Surg 2005;92: 1002-1027.

8. Saleh HA, Jackson H, Khatib G, Banerjee M. Correlation of bcl-2 on coprotein immunohisto chemical expression with proliferation index and histopathologic parameters in colorectal neoplasia. Pathol Oncol Res 1999;5:273-279.

9. Georgescu CV, Săftoiu A, Georgescu CC, Ciurea R, Ciurea T. Correlations of proliferation markers, p53 expression and histological findings in colorectal carcinoma. J Gastrointestin Liver Dis 2007;16:133-139.

10. Ahmed NY, Ismail AT, Kareem TS. A clinicopathologic study of Ki-67 proliferation index in colorectal carcinoma. Saudi Med J 2012;33(8):841-5.

11. Ishida $H$, Sadahiro $S$, Suzuki T, Ishikawa K, Kamijo A, Tajima $T$, et al. Proliferative, infiltrative, and metastatic activities in colorectal tumors assessed by MIB-1 antibody. Oncol Rep 2003;10:1741-1745.
12. Lanza G. Jr, Cavazzini L, Borghi L, Ferretti S, Buccoliero F, Rubbini M. Immunohistochemical assessment of growth fractions in colorectal adenocarcinomas with monoclonal antibody Ki-67. Relation to clinical and pathological variables. Pathol Res Pract 1990;186:608-618.

13. Jansson A, Sun XF. Ki-67 expression in relation to clinicopathological variables and prognosis in colorectal adenocarcinomas. APMIS 1997;9730-4.

14. Kyzer S, Gordon PH. Determination of proliferative activity in colorectal carcinoma using monoclonal antibody Ki67. Dis Colon Rectum 1997;40:322-325.

15. Sahin AA, Ro JY, Brown RW, Ordonez NG, Cleary KR, et al. Assessment of Ki-67-derived tumor proliferative activity in colorectal adenocarcinomas. Mod Pathol 1994;7:17-22.

16. Melling N, Kowitz CM, Simon R, et al. High Ki67 expression is an independent good prognostic marker in colorectal cancer. J Clin Pathol 2016;69:209-214.doi:10.1136/ jclinpath-2015-202985

17. Petrowsky H, Sturm I, Graubitz O, Kooby DA, Staib-Sebler $\mathrm{E}$, Gog C, et al. Relevance of Ki-67 antigen expression and $\mathrm{K}$-ras mutation in colorectal liver metastases. Eur J Surg Oncol 2001;27:80-87

18. Michael-Robinson JM, Reid LE, Purdie DM, BiemerHüttmann $A E$, Walsh MD, Pandeya $N$, et al. Proliferation, apoptosis, and survival in high-level microsatellite instability sporadic colorectal cancer. Clin Cancer Res $2001 ; 7: 2347-2356$. 